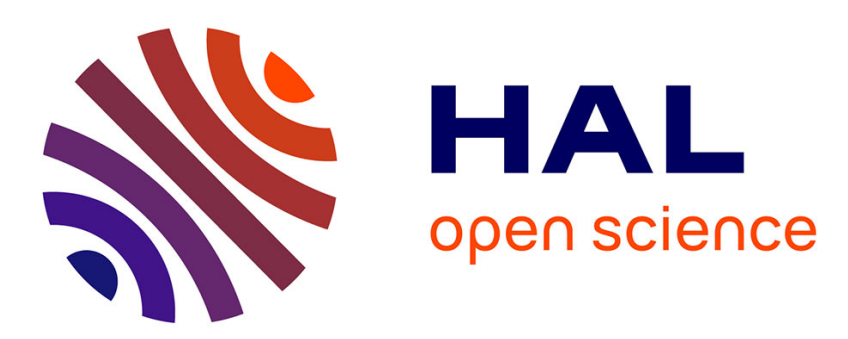

\title{
Nonlocal Estimation of Manifold Structure
}

\author{
Yoshua Bengio, Martin Monperrus, Hugo Larochelle
}

\section{To cite this version:}

Yoshua Bengio, Martin Monperrus, Hugo Larochelle. Nonlocal Estimation of Manifold Structure. Neural Computation, 2006, 18 (10), pp.2509-2528. 10.1162/neco.2006.18.10.2509 . hal-01575345

\section{HAL Id: hal-01575345 \\ https://hal.science/hal-01575345}

Submitted on 21 Oct 2018

HAL is a multi-disciplinary open access archive for the deposit and dissemination of scientific research documents, whether they are published or not. The documents may come from teaching and research institutions in France or abroad, or from public or private research centers.
L'archive ouverte pluridisciplinaire HAL, est destinée au dépôt et à la diffusion de documents scientifiques de niveau recherche, publiés ou non, émanant des établissements d'enseignement et de recherche français ou étrangers, des laboratoires publics ou privés. 


\title{
Non-Local Estimation of Manifold Structure
}

\section{Yoshua Bengio, Martin Monperrus and Hugo Larochelle}

Département d'Informatique et Recherche Opérationnelle

Centre de Recherches Mathématiques

Université de Montréal

Montréal, Québec, Canada, H3C 3J7

\{bengioy,larocheh\}@iro.umontreal.ca,martin.monperrus@laposte.net

http://www.iro.umontreal.ca/ bengioy

\section{Revised Manuscript for Neural Computation}

May 9, 2006

\begin{abstract}
We claim and present arguments to the effect that a large class of manifold learning algorithms that are essentially local and can be framed as kernel learning algorithms will suffer from the curse of dimensionality, at
\end{abstract}


the dimension of the true underlying manifold. This observation suggests to explore non-local manifold learning algorithms which attempt to discover shared structure in the tangent planes at different positions. A criterion for such an algorithm is proposed and experiments estimating a tangent plane prediction function are presented, showing its advantages with respect to local manifold learning algorithms: it is able to generalize very far from training data (on learning handwritten character image rotations), where a local non-parametric method fails.

\section{Introduction}

A central issue in order to obtain generalization is how information from the training examples are used to make predictions about new examples. In non-parametric models there are no strong prior assumptions about the structure of the underlying generating distribution, and this might make it difficult to generalize far from the training examples, as illustrated by the curse of dimensionality. There has been in recent years a lot of work on unsupervised learning based on characterizing a possibly non-linear manifold near which the data would lie, such as Locally Linear

Embedding (LLE) (Roweis and Saul, 2000), Isomap (Tenenbaum, de Silva and Langford, 2000), kernel Principal Component Analysis (PCA) (Schölkopf, Smola and Müller, 1998), Laplacian Eigenmaps (Belkin and Niyogi, 2003), and Manifold Charting (Brand, 2003). These are all essentially non-parametric methods that 
can be shown to be kernel methods with an adaptive kernel (Bengio et al., 2004), and which represent the manifold on the basis of local neighborhood relations, very often constructed using the nearest neighbors graph (the graph with one vertex per observed example, and arcs between near neighbors). The above methods characterize the manifold through an embedding which associates each training example (an input object) with a low-dimensional coordinate vector (the coordinates on the manifold). Other closely related methods characterize the manifold as well as "noise" around it. Most of these methods consider the density as a mixture of flattened Gaussians, e.g. mixtures of factor analyzers (Ghahramani and Hinton, 1996), Manifold Parzen windows (Vincent and Bengio, 2003), and other Local PCA models such as mixtures of probabilistic PCA (Tipping and Bishop, 1999). This is not an exhaustive list, and recent work also combines modeling through a mixture density and dimensionality reduction (Teh and Roweis, 2003; Brand, 2003).

In this paper we claim that there is a fundamental weakness with such nonparametric kernel methods, due to the locality of learning: we show that the local tangent plane of the manifold at a point $x$ is defined based mostly on the near neighbors of $x$ according to some possibly data-dependent kernel $K_{D}$. As a consequence, it is difficult with such methods to generalize to new combinations of values $x$ that are "far" from the training examples $x_{i}$, where being "far" is a notion that should be understood in the context of several factors: the amount of 
noise around the manifold (the examples do not lie exactly on the manifold), the curvature of the manifold, and the dimensionality of the manifold. For example, if the manifold curves quickly around $x$, neighbors need to be closer for a locally linear approximation to be meaningful, which means that more data are needed. Dimensionality of the manifold compounds that problem because the amount of data thus needed will grow exponentially with it. Saying that $y$ is "far" from $x$ means that $y$ is not well represented by its projection on the tangent plane at $x$. In this paper we explore one way to address that problem, based on estimating the tangent planes of the manifolds as a function of $x$, with parameters that can be estimated not only from the data around $x$ but from the whole dataset. Note that there can be more than one manifold (e.g. in vision, one may imagine a different manifold for each "class" of object), but the structure of these manifolds may be related, something that many previous manifold learning methods did not take advantage of. We present experiments on a variety of tasks illustrating the weaknesses of the local manifold learning algorithms enumerated above. The most striking result is that the model is able to generalize a notion of rotation learned on one kind of image (digits) to a very different kind (alphabet characters), i.e. very far from the training data. 


\section{$2 \quad$ Local Manifold Learning}

By "local manifold learning", we mean a method that derives information about the local structure of the manifold (i.e. implicitly its tangent directions) at $x$ based mostly on the training examples "around" $\mathrm{x}$. There is a large group of manifold learning methods (as well as the spectral clustering methods) that share several characteristics, and can be seen as data-dependent kernel PCA (Bengio et al., 2004). These include LLE (Roweis and Saul, 2000), Isomap (Tenenbaum, de Silva and Langford, 2000), kernel PCA (Schölkopf, Smola and Müller, 1998) and Laplacian Eigenmaps (Belkin and Niyogi, 2003). They first build a datadependent Gram matrix $M$ with $n \times n$ entries $K_{D}\left(x_{i}, x_{j}\right)$ where $D=\left\{x_{1}, \ldots, x_{n}\right\}$ is the training set and $K_{D}$ is a data-dependent kernel, and compute the eigenvectoreigenvalue pairs $\left\{\left(v_{k}, \lambda_{k}\right)\right\}$ of $M$. The embedding of the training set is obtained directly from the principal eigenvectors $v_{k}$ of $M$ (the $i$-th element of $v_{k}$ gives the $k$ th coordinate of $x_{i}$ 's embedding, i.e. $e_{k}\left(x_{i}\right)=v_{k i}$, possibly scaled by $\left.\sqrt{\frac{\lambda_{k}}{n}}\right)$ and the embedding for a new example can be estimated using the Nyström formula (Bengio et al., 2004):

$$
e_{k}(x)=\frac{1}{\lambda_{k}} \sum_{i=1}^{n} v_{k i} K_{D}\left(x, x_{i}\right)
$$

for the $k$-th coordinate of $x$, where $\lambda_{k}$ is the $k$-th eigenvalue of $M$ (the optional

scaling by $\sqrt{\frac{\lambda_{k}}{n}}$ would also apply). The above equation says that the embedding for a new example $x$ is a local interpolation of the manifold coordinates of its 
neighbors $x_{i}$, with interpolating weights given by $\frac{K_{D}\left(x, x_{i}\right)}{\lambda_{k}}$. To see more clearly how the tangent plane may depend only on the neighbors of $x$, consider the relation between the tangent plane and the embedding function: the tangent plane at $x$ is simply the subspace spanned by the vectors $\frac{\partial e_{k}(x)}{\partial x}$, as illustrated in Figure 1. It can be shown that in the case of very "local" kernels like that of LLE, spectral clustering with Gaussian kernel, Laplacian Eigenmaps or kernel PCA with Gaussian kernel, that derivative only depends significantly on the near neighbors of $x$. Consider first the simplest case, kernel PCA with a Gaussian kernel: then $\frac{\partial e_{k}(x)}{\partial x}$ can be closely approximated by a linear combination of the difference vectors $\left(x-x_{j}\right)$ for $x_{j}$ near $x$. The weights of that combination may depend on the whole data set, but if the ambiant space has many more dimensions then the number of such "near" neighbors of $x$, this is a very strong locally determined constraint on the shape of the manifold.

Let us now consider the case of LLE. A kernel consistent with LLE is $K_{L L E}\left(x, x_{i}\right)$ being the weight of $x_{i}$ in the reconstruction of $x$ by its $k$ nearest neighbors (Bengio et al., 2004). This weight is obtained by the following equation (Saul and Roweis, 2002):

$$
K_{L L E}\left(x, x_{i}\right)=\frac{\sum_{j=1}^{k} G_{i j}^{-1}}{\sum_{l, m=1}^{k} G_{l m}^{-1}}
$$

with $G^{-1}$ the inverse of the local Gram matrix $G$

$$
G_{l m}=\left(x-x_{l}\right) \cdot\left(x-x_{m}\right)
$$


for all pairs $\left(x_{l}, x_{m}\right)$ of $k$ nearest neighbors of $x$ in the training set. Because $G^{-1}=|G|^{-1} C^{T}$ with $C$ the cofactor matrix of $G$, eq. 2 can be rewritten as

$$
K_{L L E}\left(x, x_{i}\right)=\frac{\sum_{j} s_{j} \prod_{l, m}\left(G_{l m}\right)^{t_{j l m}}}{\sum_{j} u_{j} \prod_{l, m}\left(G_{l m}\right)^{v_{j l m}}}
$$

and consequently, thanks to the usual derivation rules, its derivative is a linear combination of derivatives of terms of the form $\left(G_{l m}\right)^{t}$. But

$$
\begin{aligned}
\frac{\partial\left(G_{l m}\right)^{t}}{\partial x} & =\frac{\partial\left(\left(x-x_{l}\right) \cdot\left(x-x_{m}\right)\right)^{t}}{\partial x} \\
& =t\left(G_{l m}\right)^{t-1}\left(x-x_{l}+x-x_{m}\right)
\end{aligned}
$$

which implies that the derivative of $K_{L L E}\left(x, x_{i}\right)$ w.r.t $x$ is in the span of the vectors $\left(x-x_{j}\right)$ with $x_{j}$ one of the $k$ nearest neighbors of $x$.

The case of Isomap is less intuitively obvious but we show below that it is also local. Let $\mathcal{D}(a, b)$ denote the graph geodesic distance going only through $a, b$ and points from the training set. As shown in (Bengio et al., 2004), the corresponding data-dependent kernel can be defined as

$$
K_{D}\left(x, x_{i}\right)=-\frac{1}{2}\left(\mathcal{D}\left(x, x_{i}\right)^{2}-\frac{1}{n} \sum_{j} \mathcal{D}\left(x, x_{j}\right)^{2}-\overline{\mathcal{D}}_{i}+\overline{\mathcal{D}}\right)
$$

where

$$
\overline{\mathcal{D}}_{i}=\frac{1}{n} \sum_{j} \mathcal{D}\left(x_{i}, x_{j}\right)^{2}
$$


and

$$
\overline{\mathcal{D}}=\frac{1}{n} \sum_{j} \overline{\mathcal{D}}_{j}
$$

Let $\mathcal{N}\left(x, x_{i}\right)$ denote the index $j$ of the training set example $x_{j}$ that is the neighbor of $x$ minimizing $\left\|x-x_{j}\right\|+\mathcal{D}\left(x_{j}, x_{i}\right)$. Then

$$
\frac{\partial e_{k}(x)}{\partial x}=\frac{1}{\lambda_{k}} \sum_{i} v_{k i}\left(\frac{1}{n} \sum_{j} \mathcal{D}\left(x, x_{j}\right) \frac{\left(x-x_{\mathcal{N}\left(x, x_{j}\right)}\right)}{\left\|x-x_{\mathcal{N}\left(x, x_{j}\right)}\right\|}-\mathcal{D}\left(x, x_{i}\right) \frac{\left(x-x_{\mathcal{N}\left(x, x_{i}\right)}\right)}{\left\|x-x_{\mathcal{N}\left(x, x_{i}\right)}\right\|}\right)
$$

which is a linear combination of vectors $\left(x-x_{k}\right)$, where $x_{k}$ is a neighbor of $x$. This clearly shows that the tangent plane at $x$ associated with Isomap is also included in the subspace spanned by the vectors $\left(x-x_{k}\right)$ where $x_{k}$ is a neighbor of $x$.

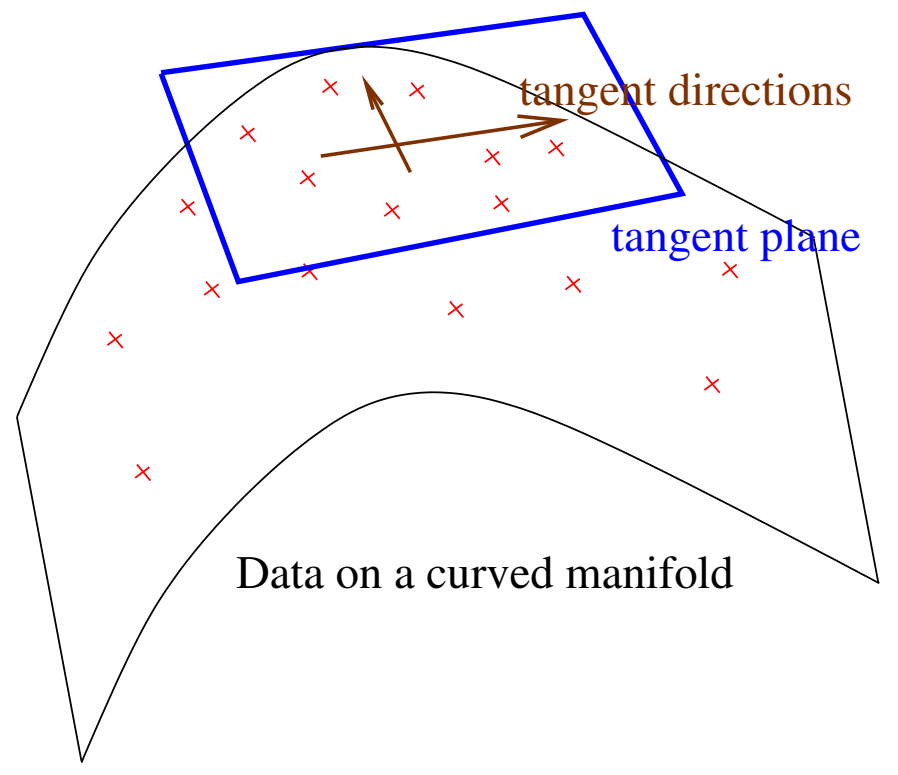

Figure 1: The tangent plane is spanned by the vectors $\frac{\partial e_{k}(x)}{\partial x}$, i.e. the directions of most rapid change of coordinate $k$ when moving along the manifold 
There are also a variety of local manifold learning algorithms which can be classified as "mixtures of pancakes" (Ghahramani and Hinton, 1996; Tipping and Bishop, 1999; Vincent and Bengio, 2003; Teh and Roweis, 2003; Brand, 2003). These are generally mixtures of Gaussians with a particular covariance structure. When the covariance matrix is approximated using its principal eigenvectors, this leads to "Local PCA" types of methods. For these methods the local tangent directions directly correspond to the principal eigenvectors of the local covariance matrices. Learning is also local since it is mostly the examples around the Gaussian center that determine its covariance structure. The problem is not so much due to the form of the density as a mixture of Gaussians. The problem is that the local parameters (e.g. local principal directions) are estimated mostly based on local data. There is usually a non-local interaction between the different Gaussians, but its role is mainly of global coordination, e.g. where to set the Gaussian centers to allocate them properly where there is data, and optionally how to orient the principal directions so as to obtain a globally coherent coordinate system for embedding the data.

\subsection{Where Local Manifold Learning Would Fail}

It is easy to imagine at least four failure causes for local manifold learning methods, and combining them will create even greater problems: 


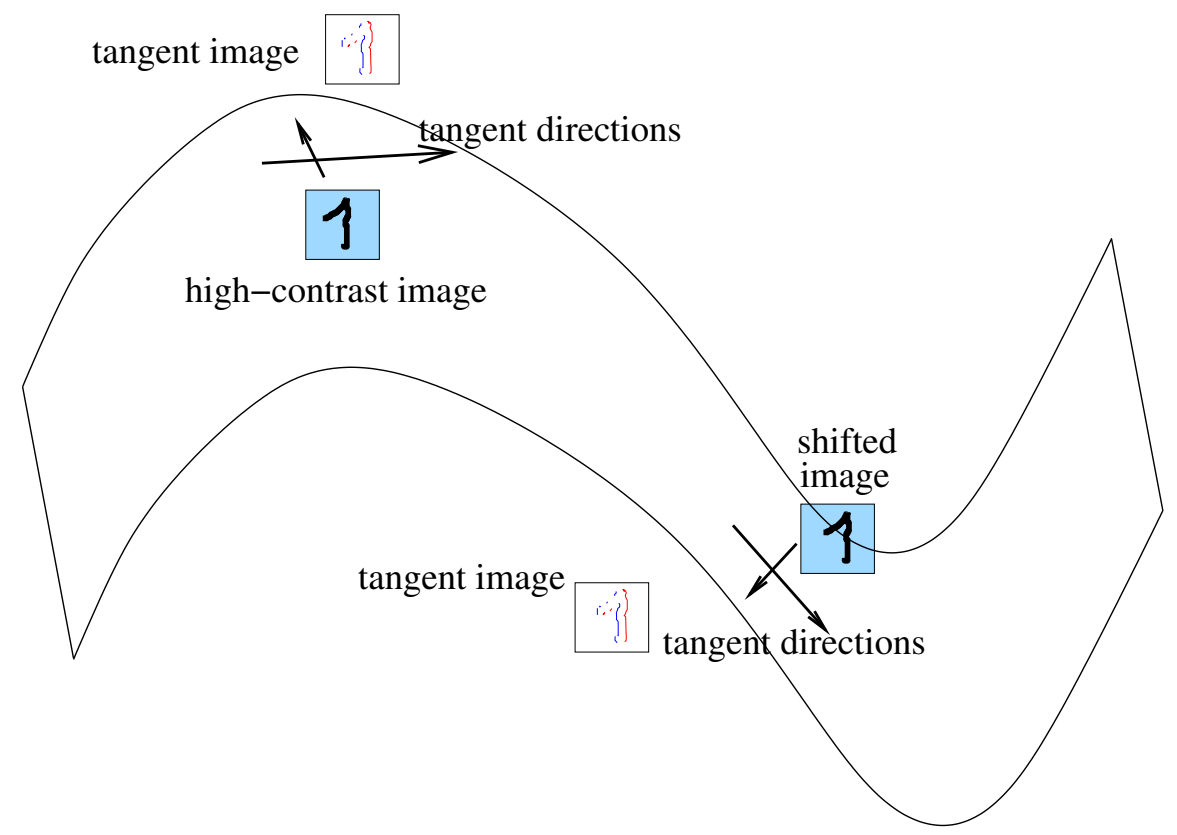

Figure 2: The manifold of translations of a high-contrast image has very high curvature. The tangent plane for an image translated by only one pixel looks similar but changes abruptly since the edges are only one-pixel wide and are also shifted by one pixel. Hence the two tangent planes are almost orthogonal.

- Noise around the manifold. Data are not exactly lying on the manifold. In the case of non-linear manifolds, the presence of noise means that more data around each pancake region will be needed to properly estimate the tangent directions of the manifold in that region. More data is needed simply to sufficiently average out the noise (i.e. some random directions quite different from the local principal directions might otherwise be selected).

- High curvature of the manifold. Local manifold learning methods basically approximate the manifold by the union of many locally linear patches. 
For this to work, there must be at least $d$ close enough examples in each patch (more with noise). With a high curvature manifold, more - smaller - patches will be needed, and the number of required patches will grow exponentially with the dimensionality of the manifold. Consider for example the manifold of translations of a high-contrast image (fig. 2). The tangent direction corresponds to the change in image due a small translation, i.e. it is non-zero only at edges in the image. After a one-pixel translation, the edges have moved by one pixel, and may not overlap much with the edges of the original image if it had high contrast. This is indeed a very high curvature manifold. In addition, if the image resolution is increased, then many more training images will be needed to capture the curvature around the translation manifold with locally linear patches. Yet the physical phenomenon responsible for translation is expressed by a simple equation, which does not get more complicated with increasing resolution.

- High intrinsic dimension of the manifold. We have already seen that high manifold dimensionality $d$ is hurtful because $O(d)$ examples are required in each patch and $O\left(r^{d}\right)$ patches (for some $r$ depending on curvature) are necessary to span the manifold.

- Presence of many manifolds with little data per manifold. In many real-world settings there is not just one global manifold but a large num- 
ber of (generally non-intersecting) manifolds which however share something about their structure. A simple example is the manifold of transformations (view-point, position, lighting,...) of 3D objects in 2D images. There is one manifold per object instance (corresponding to the successive application of small amounts of all of these transformations). If there are only a few examples for each such class then it is almost impossible to learn the manifold structures using only local manifold learning. However, if the manifold structures are generated by a common underlying phenomenon then a nonlocal manifold learning method could potentially learn all of these manifolds and even generalize to manifolds for which a single instance is observed, as demonstrated in the experiments in section 5 .

\section{Non-Local Manifold Tangent Learning}

Here we choose to characterize the manifolds in the data distribution through a matrix-valued function $F(x)$ that predicts at $x \in \mathbf{R}^{m}$ a basis for the tangent plane of the manifold near $x$, hence $F(x) \in \mathbf{R}^{d \times m}$ for a $d$-dimensional manifold. Basically, $F(x)$ specifies in which directions (w.r.t. $x$ ) one expects to find near neighbors of $x$.

We are going to consider a simple supervised learning setting to train this function. As with Isomap, we consider that the vectors $\left(x-x_{i}\right)$ with $x_{i}$ a near neighbor of $x$ 
span a noisy estimate of the manifold tangent space. We propose to use them to define a "noisy target" for training $F(x)$. In our experiments we simply collected the $k$ nearest neighbors of each example $x$, but better selection criteria might be devised. Points on the predicted tangent subspace can be written $x+F(x)^{\prime} w$ with $w \in \mathbf{R}^{d}$ being local coordinates in the basis specified by $F(x)$. Several criteria are possible to match the neighbors differences with the subspace defined by $F(x)$. One that yields to simple analytic calculations is simply to minimize the distance between the $x-x_{j}$ vectors and their projection on the subspace defined by $F(x)$. The low-dimensional local coordinate vector $w_{t j} \in \mathbf{R}^{d}$ that matches neighbor $x_{j}$ of example $x_{t}$ is thus an extra free parameter that has to be optimized, but is obtained analytically. The overall training criterion involves a double optimization over function $F$ and local coordinates $w_{t j}$ of what we call the relative projection error:

$$
\min _{F,\left\{w_{t j}\right\}} \sum_{t} \sum_{j \in \mathcal{N}\left(x_{t}\right)} \frac{\left\|F\left(x_{t}\right)^{\prime} w_{t j}-\left(x_{t}-x_{j}\right)\right\|^{2}}{\left\|x_{t}-x_{j}\right\|^{2}}
$$

where $\mathcal{N}(x)$ denotes the selected set of near neighbors of $x$. The normalization by $\left\|x_{t}-x_{j}\right\|^{2}$ is to avoid giving more weight to the neighbors that are further away. The above ratio amounts to minimizing the square of the sinus of the projection angle. To perform the above minimization, we can do coordinate descent (which guarantees convergence to a minimum), i.e. alternate changes in $F$ and changes in w's which at each step go down the total criterion. Since the minimization over the $w$ 's can be done separately for each example $x_{t}$ and neighbor $x_{j}$, it is equivalent 
to minimize

$$
\frac{\left\|F\left(x_{t}\right)^{\prime} w_{t j}-\left(x_{t}-x_{j}\right)\right\|^{2}}{\left\|x_{t}-x_{j}\right\|^{2}}
$$

over vector $w_{t j}$ for each such pair (done analytically) and compute the gradient of the above over $F$ (or its parameters) to move $F$ slightly (we used stochastic gradient on the parameters of $F$ ). The solution for $w_{t j}$ is obtained by solving the linear system

$$
F\left(x_{t}\right) F\left(x_{t}\right)^{\prime} w_{t j}=F\left(x_{t}\right) \frac{\left(x_{t}-x_{j}\right)}{\left\|x_{t}-x_{j}\right\|^{2}} .
$$

In our implementation this is done robustly through a singular value decomposition

$$
F\left(x_{t}\right)^{\prime}=U S V^{\prime}
$$

and

$$
w_{t j}=B\left(x_{t}-x_{j}\right)
$$

where $B$ can be precomputed for all the neighbors of $x_{t}$ :

$$
B=\left(\sum_{k=1}^{d} 1_{S_{k}>\epsilon} V_{\cdot k} V_{\cdot k}^{\prime} / S_{k}^{2}\right) F\left(x_{t}\right)
$$

with $\epsilon$ a small regularization threshold. The gradient of the criterion with respect 
to the $i$-th row of $F\left(x_{t}\right)$, holding the local coordinates $w_{t j}$ fixed, is simply

$$
2 \sum_{j \in \mathcal{N}\left(x_{t}\right)} \frac{w_{t j i}}{\left\|x_{t}-x_{j}\right\|}\left(F\left(x_{t}\right)^{\prime} w_{t j}-\left(x_{t}-x_{j}\right)\right)
$$

where $w_{t j i}$ is the $i$-th element of $w_{t j}$. In practice, it is not necessary to store more than one $w_{t j}$ vector at a time. In the experiments, $F(\cdot)$ is parameterized as a an ordinary one hidden layer neural network with $m$ inputs and $d \times m$ outputs. It is trained by stochastic gradient descent, one example $x_{t}$ at a time. The rows of $F\left(x_{t}\right)$ are not constrained to be orthogonal nor to have norm 1 . They are only used to define a basis for the tangent plane.

Although the above algorithm provides a characterization of the manifold, it does not directly provide an embedding nor a density function. However, once the tangent plane function is trained, there are ways to use it to obtain all of the above. The simplest method is to apply existing algorithms that provide both an embedding and a density function based on a Gaussian mixture with pancake-like covariances. For example one could use (Brand, 2003), and the local covariance matrix around $x$ could be of the form $F(x)^{\prime} \operatorname{diag}\left(\sigma^{2}(x)\right) F(x)$, where $\sigma_{i}^{2}(x)$ should estimate $\operatorname{Var}\left(w_{i}\right)$ around $x$.

Figure 3 illustrates why Non-Local Tangent Learning can be a more accurate predictor of the tangent plane. Since the tangent plane is estimated by a smooth predictor (in our case a neural net) that has the potential to generalize non-locally, 

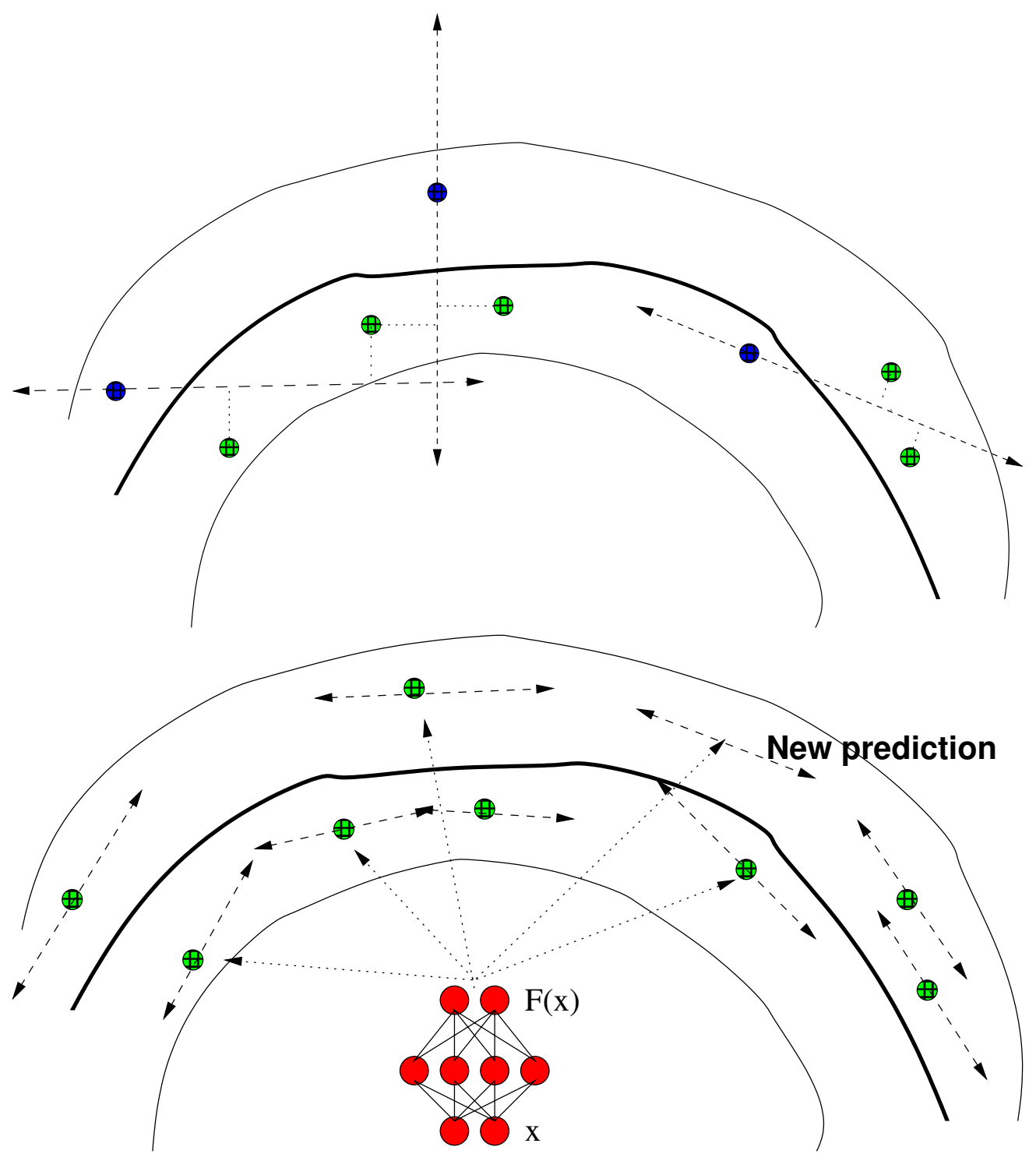

Figure 3: This figure displays the difference between local PCA and Non-Local Tangent Learning. The top image shows what the one-dimensional tangent plane learned by local PCA (using 2 nearest neighbors) might look like, for the data points in blue. The bottom image shows the same, but for Non-Local Tangent Learning. We emphasize here that with Non-Local Tangent Learning, the predicted tangent plane should change smoothly between points and new predictions 16 can be made anywhere in the data space. 
the tangent plane tends to vary smoothly between training points. This will not be true for local PCA for example, especially if there are not many training points. Note that this type of estimator can make predictions anywhere in the data space, even far from the training examples, which can be problematic for algorithms such as Local PCA.

\section{Previous Work on Manifold Learning}

The non-local manifold learning algorithm presented here (find $F(\cdot)$ which minimizes the criterion in eq. 4) is similar to the one proposed in (Rao and Ruderman, 1999) to estimate the generator matrix of a Lie group. That group defines a onedimensional manifold generated by following the orbit $x(t)=e^{G t} x(0)$, where $G$ is an $m \times m$ matrix and $t$ is a scalar manifold coordinate. A multi-dimensional manifold can be obtained by replacing $G t$ above by a linear combination of multiple generating matrices. In (Rao and Ruderman, 1999) the matrix exponential is approximated to first order by $(I+G t)$, and the authors estimate $G$ for a simple signal undergoing translations, using as a criterion the minimization of $\sum_{x, \tilde{x}} \min _{t}\|(I+G t) x-\tilde{x}\|^{2}$, where $\tilde{x}$ is a neighbor of $x$ in the data. Note that in this model the tangent plane is a linear function of $x$, i.e. $F(x)=G x$. By minimizing the above across many pairs of examples, a good estimate of $G$ for the artificial data was recovered by (Rao and Ruderman, 1999). Our proposal extends 
this approach to multiple dimensions and non-linear relations between $x$ and the tangent planes.

The work on Tangent Distance (Simard, LeCun and Denker, 1993), though more focussed on character recognition, also uses information from the tangent plane of the data manifold. In (Simard, LeCun and Denker, 1993), the tangent planes are used to build a nearest neighbor classifier that is based on the distance between the tangent subspaces around two examples to be compared. The tangent vectors that span the tangent space are not learned, but are rather obtained a priori analytically, for transformations that locally do not change the class label, such as rotation, location shift and thickness change. In (Hastie, Simard and Sackinger, 1995; Hastie and Simard, 1998), the authors present a Tangenet Subspace Learning algorithm to learn character prototypes along with a tangent plane around each prototype, which reduces the time and memory requirements of the nearest-neighbor Tangent Distance classifier. Unlike in (Rao and Ruderman, 1999), the manifold can be more than one-dimensional (they present results for 12 dimensions), but the manifold is locally linear around each prototype (hence must be globally smooth if the number of prototypes is significantly less than the number of examples). This learning procedure exploits the a priori tangent vector basis for the training points, which is computed analytically as in Tangent Distance (Simard, LeCun and Denker, 1993). Non-Local Tangent Learning can be viewed as an extension of these ideas that allows to apply them beyond the realm of image processing, avoiding the need 
for explicit prior knowledge on the invariances of objects in each class, and that also introduces the notion of non-local learning of the manifold structure.

More generally, we can say that No-Local Tangent Learning, Local PCA, LLE, Isomap and Tangent Subspace Learning all try to learn a manifold structure (either the embedding or the tangent plane) in order to respect local metric structure, but in different ways, and using different sources of information. Since all of them implicitly or explicity estimate the tangent plane, they all have the potential to learn invariants that could be useful for transformation-invariant classification. Local PCA and LLE are based on the Euclidean metric, Isomap on an approximate geodesic metric, and (Hastie, Simard and Sackinger, 1995) uses the Tangent Distance metric, based on a priori knowledge about characters. One important difference with the ideas presented here is that for all these algorithms the predicted manifold structure at $x$ is obtained essentially using only local information in the neighborhood of $x$. We believe that the main conceptual advantage of the approach proposed here over local manifold learning is that the parameters of the tangent plane predictor can be estimated using data from very different regions of space, thus in principle allowing to be less sensitive to all four of the problems described in 2.1, thanks to sharing of information across these different regions. 


\section{$5 \quad$ Experimental Results}

The objective of the experiments is to validate the proposed algorithm: does it estimate well the true tangent planes? Does it learn better than a local manifold learning algorithm?

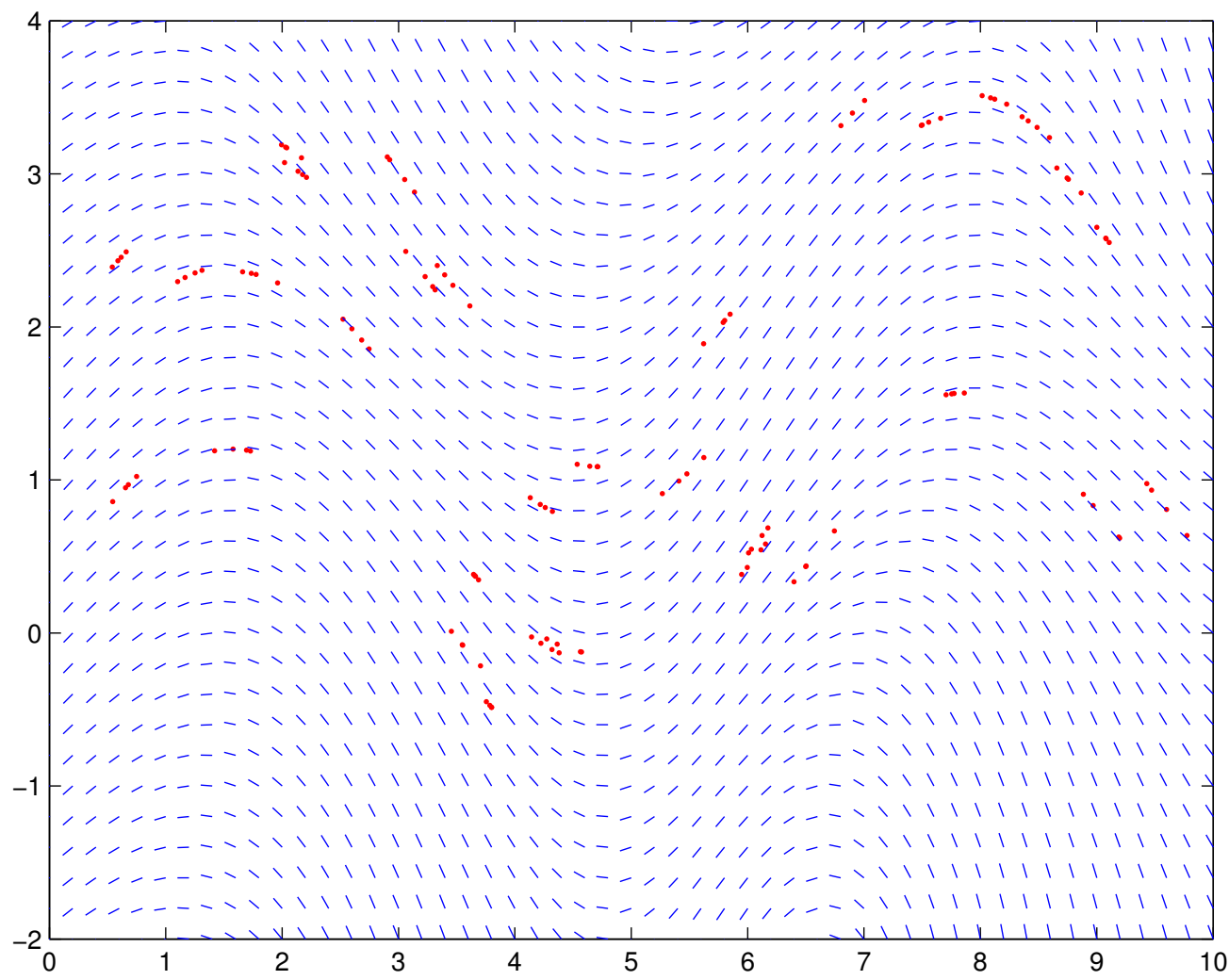

Figure 4: Task 1. 2-D data with 1-D sinusoidal manifolds: the method indeed captures the tangent planes. The small segments are the estimated tangent planes.

Small dots are the training examples. 


\subsection{Error Measurement}

In addition to visualizing the results for the low-dimensional data, we measure performance by considering how well the algorithm learns the local tangent distance, as measured by the normalized projection error of nearest neighbors (eq. 5). We compare the errors of four algorithms, always on test data not used to estimate the tangent plane: (a) true analytic (using the true manifold's tangent plane at $x$ computed analytically), (b) tangent learning (using the neural-network tangent plane predictor $F(x)$, trained using the $k \geq d$ nearest neighbors in the training set of each training set example), (c) Isomap (using the tangent plane defined in Eq. 3), (d) Local PCA (using the $d$ principal components of the empirical covariance of the $k$ nearest neighbors of $x$ in the training set).

\subsection{Tasks}

- Task 1. We first consider a low-dimensional but multi-manifold problem. The data $\left\{x_{i}\right\}$ are in 2 dimensions and coming from a set of 40 1-dimensional manifolds. Each manifold is composed of 4 near points obtained from a randomly based sinus, i.e $\forall i \in 1 . .4$,

$$
x_{i}=\left(a+t_{i}, \sin \left(a+t_{i}\right)+b\right)
$$


where $a, b$, and $t_{i}$ are randomly chosen. Four neighbors were used for training both the Non-Local Tangent Learning algorithm and the benchmark local non-parametric estimator (Local PCA of the 4 neighbors). Figure 4 shows the training set and the tangent planes recovered with Non-Local Tangent Learning, both on the training examples and generalizing away from the data. The neural network has 10 (chosen arbitrarily) hidden units. This problem is particularly difficult for local manifold learning, which does very poorly here: the out-of-sample relative projection error are respectively 0.09 for the true analytic plane, 0.25 for non-local tangent learning, and 0.81 for local PCA.

- Task 2. This is a higher dimensional manifold learning problem, with 41 dimensions. The data are generated by sampling Gaussian curves. Each curve is of the form $x(i)=e^{t_{1}-(-2+i / 10)^{2} / t_{2}}$ with $i \in\{0,1, \ldots, 40\}$. Note that the tangent vectors are not linear in $x$. The manifold coordinates are $t_{1}$ and $t_{2}$, sampled uniformly, respectively from $(-1,1)$ and $(0.1,3.1)$. Normal noise (standard deviation $=0.001)$ is added to each point. 100 example curves were generated for training and 200 for testing. The neural network has 100 hidden units. Figure 5 shows the relative projection error for the four methods on this task, for the $k$-th nearest neighbor, for increasing values of $k$. First, the error decreases because of the effect of noise (near noisy neighbors may form a high angle with the tangent plane). Then, it increases 


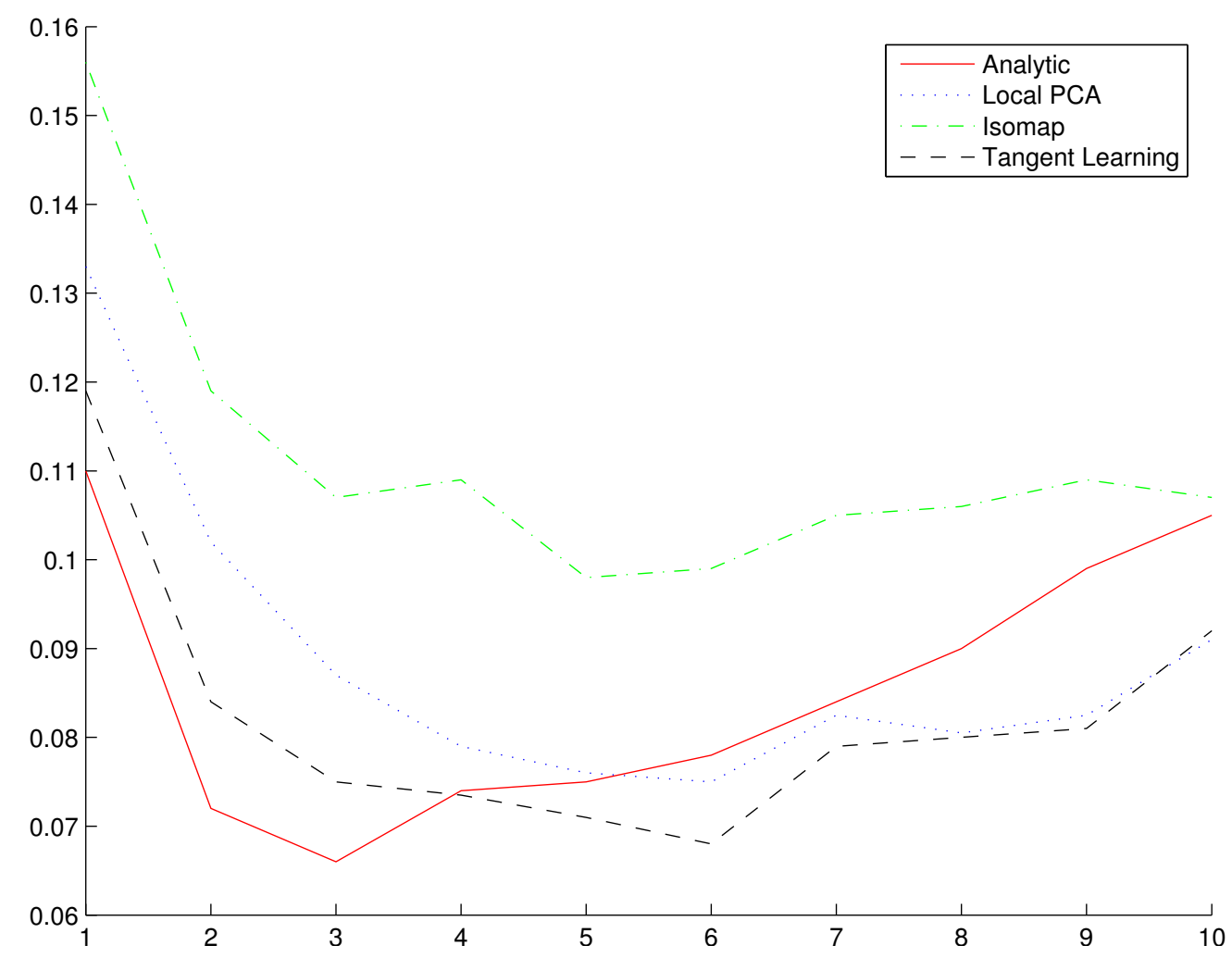

Figure 5: Task 2. Relative projection error for $k$-th nearest neighbor, w.r.t. $k$, for compared methods (from lowest to highest at $\mathrm{k}=1$ : analytic, tangent learning, local PCA, Isomap). Note U-shape due to opposing effects of curvature and noise. 


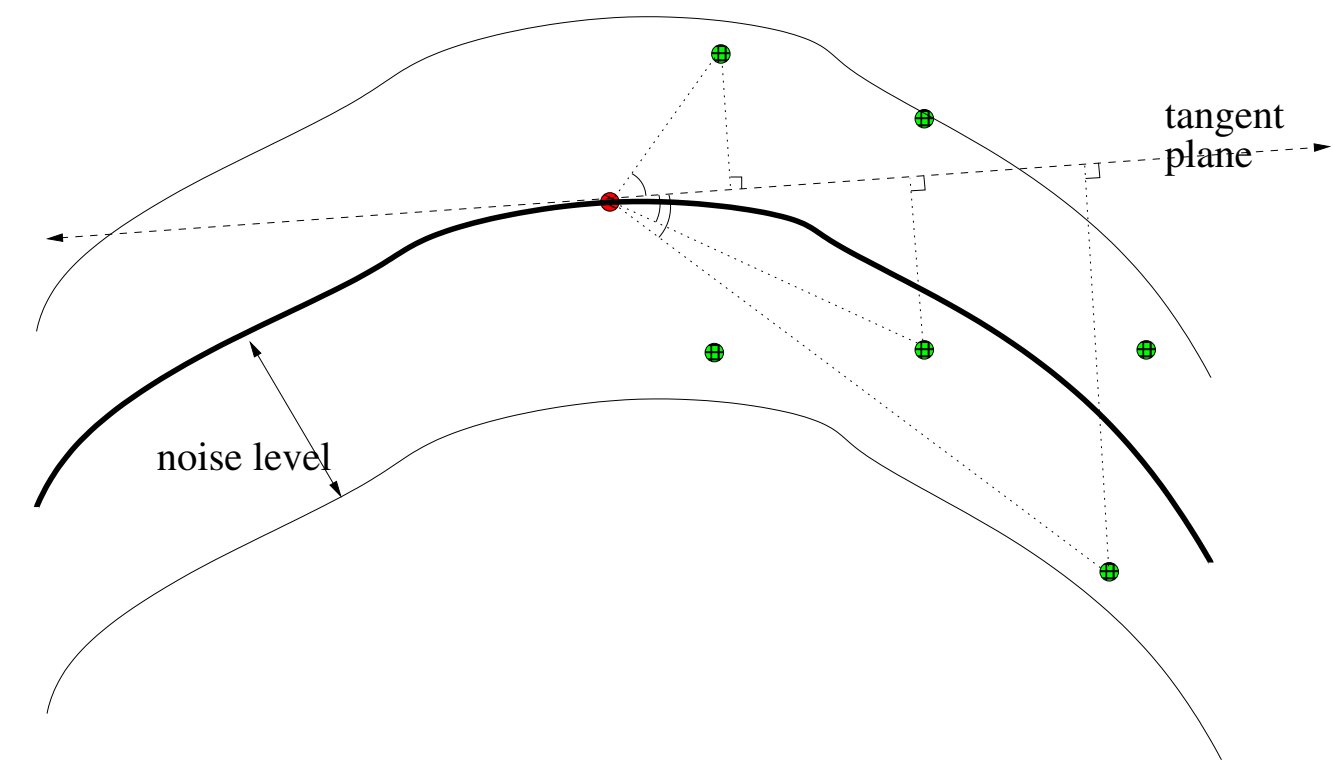

Figure 6: Schematic explanation of the U-shaped curve in projection error. With noise around manifold, nearest examples tend to have a large angle, but because of curvature the error also increases with distance to the reference point.

because of the curvature of manifold (further away neighbors form a larger angle). This effect is illustrated schematically in Figure 6, and gives rise to the U-shaped projection error curve in Figure 5.

- Task 3. This is a high-dimensional multi-manifold task, involving digit images to which we have applied slight rotations, in such a way as to have the knowledge of the analytic formulation of the manifolds. There is one rotation manifold for each instance of digit from the database, but only two examples for each manifold: one real image from the MNIST dataset and one slightly rotated image. $1000 \times 2$ examples are used for training and $1000 \times 2$ 
for testing. In this context we use $k=1$ nearest neighbor only and manifold dimension is 1 . The average relative projection error for the nearest neighbor is 0.27 for the analytic tangent plane (obtained using the same technique as in (Simard, LeCun and Denker, 1993)), 0.43 with tangent learning (100 hidden units), and 1.5 with Local PCA. Note the neural network would probably overfit if trained too much (here only 100 epochs).

An even more interesting experiment consists in applying the above trained predictor on novel images that come from a very different distribution but one that shares the same manifold structure: it was applied to images of other characters that are not digits. We have used the predicted tangent planes to follow the manifold by small steps (this is very easy to do in the case of a one-dimensional manifold). More formally, this corresponds to the following pseudo-code:

$$
\begin{aligned}
& \text { WalkOnManifold }(x, F(\cdot), i, \text { nsteps, stepsize) } \\
& \text { for } s \text { from } 1 \text { to nsteps } \\
& \begin{array}{cl}
1 . & d_{s}=S V D(F(x), i) \\
2 . & x=x+d_{s} \cdot \text { stepsize }
\end{array}
\end{aligned}
$$

where $x$ is the initial image, $F(\cdot)$ is the tangent predictor, nsteps is the number of steps, $d$ is the $i^{\text {th }}$ orthogonal basis vector of the tangent plane given by the SVD decomposition and stepsize contols how far in the direction $d$ each step is made. Note that the sign of stepsize also determines the orientation of the walk. Also, since in the present task the dimension of the 

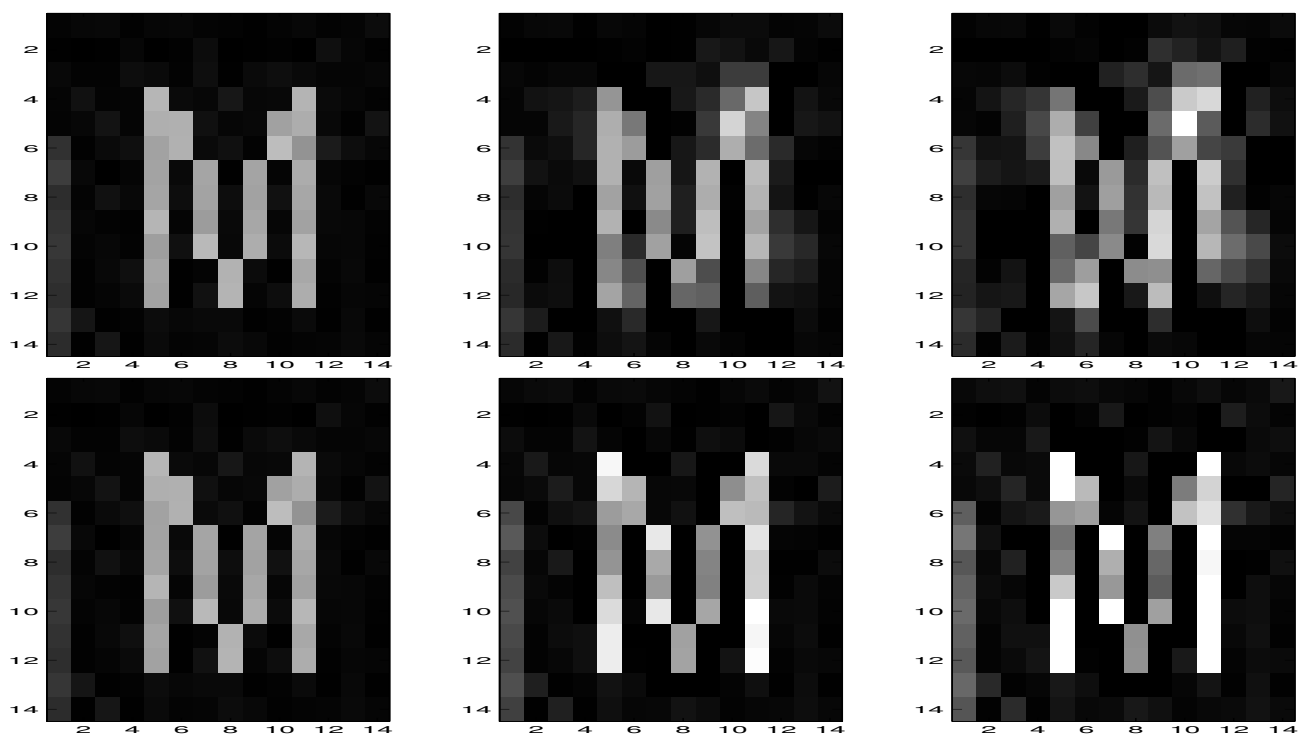

Figure 7: Left column: original image. Middle: applying a small amount of the predicted rotation. Right: applying a larger amount of the predicted rotation. Top: using the estimated tangent plane predictor. Bottom: using local PCA, which is clearly much worse (the letter is not rotated).

manifold is only 1 , then we have $i=1$ and the SVD isn't necessary. We have considered the more general case only because it will be used in the next task.

Figure 7 shows the effect of applying WalkOnManifold on a letter 'M' image for a few and a larger number of steps, both for the neural network predictor and for the local PCA predictor.

This example illustrates the crucial point that non-local tangent plane learning is able to generalize to truly novel cases, where local manifold learning 
fails. The results showed in figure 7 provide evidence of the impressive extrapolation capacity of Non-Local Tangent Learning, since the 'M' letter is quite different from any digit in the training set, i.e. the neural network is not just locally smoothing the tangent plane estimation, but it truly allows to generalize the notion of rotation (here) to new objects.

Since this experiment was set so that the only class invariant transformation that could be learned would be the rotation transformation, one might wonder in what ways this task differs from supervised learning, i.e., predicting the effect of a slight rotation on an image. First of all one should note that we are predicting an undirected vector (i.e. rotations one way or the other are both acceptable), and second the procedure can be readily generalized to predicting a whole tangent plane, without prior knowledge about invariants of the inputs, as shown with the next set of experiments, in which only natural data are used to infer the shape of the manifold.

- Task 4. Finally, we performed the following experiment in order to observe the invariances that can be learned with Non-Local Tangent Learning for a typical character recognition dataset. These invariances can be compared with those reported for other methods, such as in (Hastie, Simard and Sackinger, 1995). We used the first 6291 examples from the USPS training set to train a separate neural network per each digit class, using Non-Local Tangent Learning. For this experiment, the hyper-parameters 
(number of neighbors, number of hidden units, number of training epochs through early stopping) were tuned using a validation set (the 1000 USPS examples following the training set), with normalized projection error. The manifold dimension was arbitrarily chosen to be 7 , following inspiration from the Tangent Distance work (Simard, LeCun and Denker, 1993). Then, for each class, we chose one digit example, and performed a walk on the manifold as indicated by the pseudo-code of WalkOnManifold of task 3, in order to visualize the learned manifold around the example image.

The results are plotted in figure 8 . The values of nsteps, nsteps and $i$ where tuned manually to clarify visually the effect of the learned transformations. Note that those transformations are not linear, since the directions $d_{s}$ are likely to be different from one step to another, and visual inspection also suggests so (e.g. changing the shape of the loop in a '2').

The overall picture is rather good, and some of the digit transformations are quite impressive, showing that the model learned typical transformations. For instance, we can see that Non-Local Tangent Learning was able to rotate the digit "8" so that it would stand straight. In our opinion, those transformations compare well to those reported in (Hastie, Simard and Sackinger, 1995), although no prior knowledge about images was used here in order to obtain these transformations. 

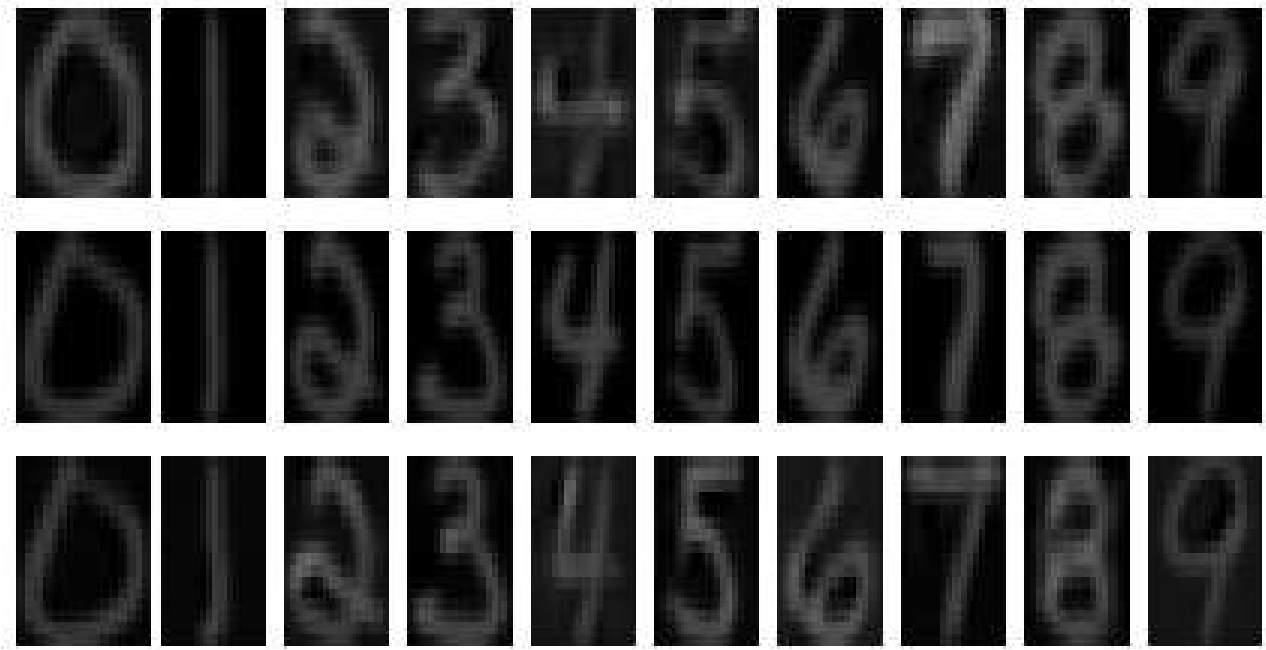

Figure 8: Task 4. Examples of the "walk on the manifold" for digit samples of USPS (middle row). There is one model per digit class (column). Moving up or down the column corresponds to moving along one of the learned directions. Only the middle row corresponds to an actual example image, the other rows are obtained by walking one way or the other along the manifold. 
In (Bengio and Larochelle, 2006), we describe an extension of Non-Local Tangent Learning, Non-Local Manifold Parzen, which uses non-local learning to train a Manifold Parzen (Vincent and Bengio, 2003) density estimator. The basic idea is to estimate not only the tangent plane but also variances in each of the local principal directions, still as functions of the tangent plane connecting point $x$. Having both principal directions and variances one can write down a locally Gaussian density and estimate the global density as a mixture of these Gaussian components (one on each training example). From the density one can readily obtain a classifier using one density estimator per class. Improvements with respect to local learning algorithms on the out-of-sample likelihood and classification error are reported for toy and real life problems, such as the USPS digit recognition task. Note that this extension provides other ways to do model selection, e.g. by cross-validation on the out-of-sample likelihood or classification error.

\section{Conclusion}

The central claim of this paper is that there are fundamental problems with nonparametric local approaches to manifold learning, essentially due to the curse of dimensionality (at the dimensionality of the manifold), but worsened by manifold curvature, noise, and the presence of several disjoint manifolds. To address these problems, we propose that learning algorithms should be designed in such 
a way that they can share information, coming from different regions of space, about the structure of the manifold. In this spirit we have proposed a simple learning algorithm based on predicting the tangent plane at $x$ with a function $F(x)$ whose parameters are estimated based on the whole data set. Note that the same fundamental problems are present with non-parametric approaches to semi-supervised learning (e.g. as in (Szummer and Jaakkola, 2002; Chapelle, Weston and Scholkopf, 2003; Belkin and Niyogi, 2003; Zhu, Ghahramani and Lafferty, 2003)), which rely on proper estimation of the manifold in order to propagate label information.

Future work should investigate how to better handle the curvature problem, e.g. by following the manifold (using the local tangent estimates), to estimate a manifoldfollowing path between pairs of neighboring examples. The algorithm was already extended to obtain a mixture of factor analyzers in (Bengio and Larochelle, 2006) (with the factors or the principal eigenvectors of the Gaussian centered at $x$ obtained from $F(x))$. This view provides an alternative criterion to optimize $F(x)$ (the local log-likelihood of such a Gaussian). This criterion also tells us how to estimate the missing information (the variances along the eigenvector directions). On the other hand, since we can estimate $F(x)$ everywhere, a more ambitious view would consider the density as a "continuous" mixture of Gaussians (with an infinitesimal component located everywhere in space). 


\section{Acknowledgments}

The authors would like to thank the following funding organizations for support: NSERC, MITACS, IRIS, and the Canada Research Chairs. They also want to thank Olivier Delalleau for helpful exchanges.

\section{References}

Belkin, M. and Niyogi, P. (2003). Using manifold structure for partially labeled classification. In Becker, S., Thrun, S., and Obermayer, K., editors, Advances in Neural Information Processing Systems 15, Cambridge, MA. MIT Press.

Bengio, Y., Delalleau, O., Le Roux, N., Paiement, J.-F., Vincent, P., and Ouimet, M. (2004). Learning eigenfunctions links spectral embedding and kernel PCA. Neural Computation, 16(10):2197-2219.

Bengio, Y. and Larochelle, H. (2006). Non-local manifold parzen windows. In Advances in Neural Information Processing Systems 18. MIT Press.

Brand, M. (2003). Charting a manifold. In Becker, S., Thrun, S., and Obermayer, K., editors, Advances in Neural Information Processing Systems 15. MIT Press.

Chapelle, O., Weston, J., and Scholkopf, B. (2003). Cluster kernels for semi-supervised learning. In Becker, S., Thrun, S., and Obermayer, K., editors, Advances in Neural Information Processing Systems 15, Cambridge, MA. MIT Press. 
Ghahramani, Z. and Hinton, G. (1996). The EM algorithm for mixtures of factor analyzers. Technical Report CRG-TR-96-1, Dpt. of Comp. Sci., Univ. of Toronto.

Hastie, T. and Simard, P. (1998). Metrics and models for handwritten character recognition. Statistical Science, 13(1):54-65.

Hastie, T., Simard, P., and Sackinger, E. (1995). Learning prototype models for tangent distance. In Tesauro, G., Touretzky, D., and Leen, T., editors, Advances in Neural Information Processing Systems 7, pages 999-1006. MIT Press.

Rao, R. and Ruderman, D. (1999). Learning lie groups for invariant visual perception. In Kearns, M., Solla, S., and Cohn, D., editors, Advances in Neural Information Processing Systems 11, pages 810-816. MIT Press, Cambridge, MA.

Roweis, S. and Saul, L. (2000). Nonlinear dimensionality reduction by locally linear embedding. Science, 290(5500):2323-2326.

Saul, L. and Roweis, S. (2002). Think globally, fit locally: unsupervised learning of low dimensional manifolds. Journal of Machine Learning Research, 4:119-155.

Schölkopf, B., Smola, A., and Müller, K.-R. (1998). Nonlinear component analysis as a kernel eigenvalue problem. Neural Computation, 10:1299-1319.

Simard, P., LeCun, Y., and Denker, J. (1993). Efficient pattern recognition using a new transformation distance. In Giles, C., Hanson, S., and Cowan, J., editors, Advances in Neural Information Processing Systems 5, pages 50-58, Denver, CO. Morgan Kaufmann, San Mateo. 
Szummer, M. and Jaakkola, T. (2002). Partially labeled classification with markov random walks. In Dietterich, T., Becker, S., and Ghahramani, Z., editors, Advances in Neural Information Processing Systems 14, Cambridge, MA. MIT Press.

Teh, Y. W. and Roweis, S. (2003). Automatic alignment of local representations. In Becker, S., Thrun, S., and Obermayer, K., editors, Advances in Neural Information Processing Systems 15. MIT Press.

Tenenbaum, J., de Silva, V., and Langford, J. (2000). A global geometric framework for nonlinear dimensionality reduction. Science, 290(5500):2319-2323.

Tipping, M. and Bishop, C. (1999). Mixtures of probabilistic principal component analysers. Neural Computation, 11(2):443-482.

Vincent, P. and Bengio, Y. (2003). Manifold parzen windows. In Becker, S., Thrun, S., and Obermayer, K., editors, Advances in Neural Information Processing Systems 15, Cambridge, MA. MIT Press.

Zhu, X., Ghahramani, Z., and Lafferty, J. (2003). Semi-supervised learning using Gaussian fields and harmonic functions. In ICML'2003. 